\title{
L1157: Interaction of the molecular outflow with the Class 0 environment
}

\author{
M. T. Beltrán ${ }^{1,2}$, F. Gueth ${ }^{3}$, S. Guilloteau ${ }^{3}$, and A. Dutrey ${ }^{4}$ \\ ${ }^{1}$ Harvard-Smithsonian Center for Astrophysics, 60 Garden Street, Cambridge, MA 02138, USA \\ 2 Osservatorio Astrofisico di Arcetri, Largo E. Fermi 5, 50125 Firenze, Italy \\ 3 Institut de Radio Astronomie Millimétrique, 300 rue de la Piscine, 38406 Saint Martin d'Hères, France \\ ${ }^{4}$ Laboratoire d'Astrophysique de l'Observatoire de Grenoble, BP 53, 38041 Grenoble, France
}

Received 28 July 2003 / Accepted 3 December 2003

\begin{abstract}
We present high angular resolution interferometric observations $\left[^{\star}\right]$ of the dust continuum at 2.7 and $1.3 \mathrm{~mm}$, and of the $\mathrm{HC}_{3} \mathrm{~N}(J=12 \rightarrow 11)$ and $\mathrm{C}^{18} \mathrm{O}(J=2 \rightarrow 1)$ emission around L1157-mm, a Class 0 object that drives a spectacular molecular outflow. The millimeter dust emission is clearly resolved into two components, a flattened compact source of $\sim 450 \times 250 \mathrm{AU}$ at $1.3 \mathrm{~mm}$, and mass $\sim 0.1 M_{\odot}$, plus an extended envelope of $\sim 3000 \mathrm{AU}$ at $1.3 \mathrm{~mm}$, and mass $\sim 1.1 M_{\odot}$. The millimeter spectral index varies throughout the region, with the lower value found toward the compact protostar, possibly indicating grain growth in the denser regions. A strong interaction between the molecular outflow and the close protostellar environment is taking place and affects the structure of the innermost parts of the envelope. This is shown by the spatial coincidence between the molecular outflow and the dust (1.3 mm continuum) and $\mathrm{HC}_{3} \mathrm{~N}$ emission: both tracers show structures associated to the edges of the outflow lobes. Basically, the global picture sketched for the Class 0 object L1157-mm by Gueth et al. (1997) is supported. We find possible evidence of infall, but we do not detect any velocity gradient indicative of a rotating circumstellar disk.
\end{abstract}

Key words. stars: individual: L1157 - stars: formation - stars: circumstellar matter - ISM: dust, extinction - ISM: molecules

\section{Introduction}

Class 0 low-mass young stellar objects are deeply embedded in circumstellar dust and gas material, and are found associated with very energetic molecular bipolar outflows. Theory outlines a scenario where a central object is surrounded by an infalling envelope that contains most of the mass (e.g. Larson 1969; Adams et al. 1987). This infalling material is accreted onto the central protostar funneled through a circumstellar disk that grows as the system evolves (e.g. Cassen \& Moosman 1981; Terebey et al. 1984; Shu et al. 1987). On the other hand, a powerful outflow is accelerated and collimated via a magnetohydrodynamic mechanism from the surface of the accretion disk (Pudritz \& Norman 1983; Heyvaerts \& Norman 1989; Shu et al. 1994; Königl \& Pudritz 2000). Infalling, outflowing and rotational motions are, thus, simultaneously taking place in extremely young protostar environments, making the morphology and kinematics of such regions very complex. Observations of the millimeter thermal dust continuum and high-density molecular tracers can, in principle, reveal the phenomena taking place in the innermost parts of dense cores (e.g. Saito et al. 1996;

Send offprint requests to: F. Gueth, e-mail: gueth@iram. fr

* Based on observations carried out with the IRAM Plateau de Bure Interferometer. IRAM is supported by INSU/CNRS (France), MPG (Germany) and IGN (Spain).
Ohashi et al. 1997, 1999; Lai \& Crutcher 2000; Hogerheijde 2001). However, the strong interaction between the outflow and the surrounding dense material, which has been actually observed near the driving source in a few cases (e.g. L1551-IRS5: Fuller et al. 1995; Ladd et al. 1995; L1157: Gueth et al. 1997; L1527: Ohashi et al. 1997; Hogerheijde et al. 1998; Motte \& André 2001), complicates this portrait. In order to get an accurate picture of the complex environment of Class 0 objects and to interpret the gas kinematics in these sources, high angular resolution interferometric observations of different tracers are definitely required.

L1157-mm is a Class 0 object located at $440 \mathrm{pc}$ with a $L_{\mathrm{bol}} \simeq 11 L_{\odot}$. It is associated with IRAS $20386+6751$ and drives a spectacular outflow. The L1157 outflow has been studied in details through many molecular lines, such as CO (Umemoto et al. 1992; Gueth et al. 1996; Bachiller \& Pérez Gutiérrez 1997; Hirano \& Taniguchi 2001), SiO (Zhang et al. 1995, 2000; Gueth et al. 1998; Bachiller et al. 2001), $\mathrm{H}_{2}$ (Hodapp 1994; Davis \& Eislöffel 1995), $\mathrm{NH}_{3}$ (Bachiller et al. 1993; Tafalla \& Bachiller 1995), or $\mathrm{CH}_{3} \mathrm{OH}$ (Bachiller et al. 1995, 2001; Avery \& Chiao 1996). Many other lines have been detected (Bachiller \& Pérez Gutiérrez 1997; Bachiller et al. 2001), making L1157 the prototype of chemically active outflows. Regarding the protostar itself, dust continuum observations have been carried out at $2.7 \mathrm{~mm}$ (Gueth et al. 1996, 1997), 
$1.3 \mathrm{~mm}$ (Shirley et al. 2000; Motte \& André 2001; Chini et al. 2001; Gueth et al. 2003), $850 \mu \mathrm{m}$ (Shirley et al. 2000; Chini et al. 2001), and $450 \mu \mathrm{m}$ (Chini et al. 2001).

In a first attempt to understand the morphology of this young object, Gueth et al. (1997) carried out ${ }^{13} \mathrm{CO}(J=1 \rightarrow 0), \mathrm{C}^{18} \mathrm{O}(J=1 \rightarrow 0)$, and $2.7 \mathrm{~mm}$ continuum IRAM Plateau de Bure interferometric observations of the region around L1157-mm, with a $\sim 2$ '. 5 angular resolution. The continuum emission clearly shows two components: a marginally resolved compact, flattened core, perpendicular to the outflow direction, and a low-level extended emission that seems to delineate the heated edges of the cavity excavated by the $\mathrm{CO}$ outflow. The ${ }^{13} \mathrm{CO}$ emission also originates from the limb-brightened edges of the outflow, whereas the $\mathrm{C}^{18} \mathrm{O}$ emission is more directly associated with the compact continuum source, and shows marginal evidence of rotation. In addition, redshifted self-absorption is present in the ${ }^{13} \mathrm{CO}$ spectrum, which suggests infall motions. However, in order to confirm the picture described by Gueth et al. (1997), higher angular resolution and higher frequency continuum observations, together with different, higher density, tracers were necessary. In this work we present these new interferometric observations of the dust continuum at 2.7 and $1.3 \mathrm{~mm}$, and of the $\mathrm{HC}_{3} \mathrm{~N}(J=12 \rightarrow 11)$ and $\mathrm{C}^{18} \mathrm{O}(J=2 \rightarrow 1)$ emission around L1157-mm. These new maps reveal important details of the structure of the central source, and of the interaction between the outflow and the high-density core.

\section{Observations}

\subsection{Interferometric data}

Observations were carried out with the IRAM Plateau de Bure Interferometer (Guilloteau et al. 1992) between November 1995 and April 1996. Five different configurations of the four-antennas array were used. The longest baseline was $288 \mathrm{~m}$ (B2) and the shortest $24 \mathrm{~m}$ (B2, D). The phase center was set to the position $\alpha(\mathrm{J} 2000)=20^{\mathrm{h}} 39^{\mathrm{m}} 06.19, \delta(\mathrm{J} 2000)=$ $68^{\circ} 02^{\prime} 15^{\prime \prime} .9$, which is the L $1157-\mathrm{mm}$ position reported by Gueth et al. (1997). The source was observed simultaneously at $109.2 \mathrm{GHz}\left(\mathrm{HC}_{3} \mathrm{~N} J=12 \rightarrow 11\right)$ in USB and $219.6 \mathrm{GHz}$ $\left(\mathrm{C}^{18} \mathrm{O} J=2 \rightarrow 1\right)$ in LSB. The spectra were analyzed using a correlator with one band of $10 \mathrm{MHz}$ centered on the $\mathrm{HC}_{3} \mathrm{~N}(J=12 \rightarrow 11)$ line, one band of $20 \mathrm{MHz}$ centered on the $\mathrm{C}^{18} \mathrm{O}(J=2 \rightarrow 1)$ line, and two bands of $160 \mathrm{MHz}$ for the 2.7 and $1.3 \mathrm{~mm}$ continuum. The spectral resolution was $0.11 \mathrm{~km} \mathrm{~s}^{-1}$ in both narrow bands. The bandpass of the receivers was calibrated by observations of the strong quasars 3C273, 3C454.3, 0923+392, or 1823+568. Amplitude and phase calibrations were achieved by monitoring $1823+568$, whose flux density was determined relative to $3 \mathrm{C} 273$ and 3C454.3.

However, the emission of $1823+568$ turned out to be linearly polarized, and therefore presented a sinusoidal dependence of the amplitude with time i.e. with parallactic angle. Special considerations had thus to be taken during the calibration process. Since the polarization of the two receivers ( 2.7 and $1.3 \mathrm{~mm}$ ) is orthogonal, the amplitude curve $A(t)$ of a linearly polarized source has an opposite behavior on both receivers. This effect is only noticeable in tracks of several hours. To fix this polarization problem, we first ran a procedure to fit the calibrator amplitude curve with a sinusoidal function of the parallactic angle $\Psi$, for each scan. The functions were proportional to $1+C 1 \times \cos ^{2}(\Psi+\Phi)$, for the $2.7 \mathrm{~mm}$ receiver, and to $1+C 2 \times \sin ^{2}(\Psi+\Phi)$ for the $1.3 \mathrm{~mm}$ receiver, where $C 1$ and $C 2$ give an estimate of the percentage of polarized flux, and $\Phi$ gives an idea of the polarization angle. Then, we calibrated the amplitude curve against one of the well-known calibrators also used in the observations (e.g. 3C273), in order to obtain the real value of the flux for each scan. Thus, instead of a constant value for the flux of the amplitude calibrator, we obtained a function of the parallactic angle $\Psi$, and this function is the one we used in the calibration process.

We estimate that the uncertainties on the absolute flux density calibration are $\sim 20 \%$ at $2.7 \mathrm{~mm}$, and $\sim 40 \%$ at $1.3 \mathrm{~mm}$, due to the difficulties encountered to calibrate the amplitude. Typical rms phase noise was better than $10^{\circ}$ at $2.7 \mathrm{~mm}$, and better than $20^{\circ}$ at $1.3 \mathrm{~mm}$. The data were calibrated and analyzed with the GILDAS software package developed at IRAM and Observatoire de Grenoble. The synthesized CLEANed beam for maps made using natural weighting was 2 '. $24 \times 22^{\prime \prime} 04$ at $\mathrm{PA}=33^{\circ}$ at $2.7 \mathrm{~mm}$, and $1^{\prime \prime} .24 \times 1^{\prime \prime} .14$ at $\mathrm{PA}=71^{\circ}$ at $1.3 \mathrm{~mm}$. Unless explicitly mentioned, we subtracted the continuum from the line emission. This was performed directly in the $u v$ plane in order to avoid non linearity effects in the deconvolution, and thus any amplification of errors induced in this process.

\subsection{Bolometer data}

Gueth et al. (2003) presented $1.3 \mathrm{~mm}$ bolometer measurements obtained with the MPIfR 19-channel bolometer array (MAMBO) on the IRAM 30-m telescope. We used this map to extract short-spacings information and thus complement the interferometric data set. The interferometer and 30-m data at $1.3 \mathrm{~mm}$ were merged in the $u v$ plane using the procedure available in the GILDAS package. The relative weights of the interferometer and single-dish data were adjusted so that the weight density of the single-dish data matches that of the shortest interferometric baselines. A multiplicative scaling factor of 0.8 was applied to the single-dish data amplitudes, in order to compensate for the fact that the single-dish observations were carried out at a central wavelength of $\sim 1.25 \mathrm{~mm}$, whereas the interferometric observations were indeed done at $1.4 \mathrm{~mm}$. Hereafter, we will refer to the combined interferometer plus single-dish $1.3 \mathrm{~mm}$ continuum data simply as " $1.3 \mathrm{~mm}$ continuum" data. The synthesized (natural weight) CLEANed beam for the resulting map, $1^{\prime \prime} .24 \times 1^{\prime \prime} .22$ at PA $=27^{\circ}$, is very similar to the beam without the bolometer data.

\section{Continuum emission}

The millimeter continuum emission towards the core of L1157 was mapped at 2.7 and $1.3 \mathrm{~mm}$. Figure 1 shows the uniformweight map of the $2.7 \mathrm{~mm}$ emission, and the natural-weight map of the $1.3 \mathrm{~mm}$ emission. Figure 2 shows the superposition of the integrated ${ }^{12} \mathrm{CO}(J=1 \rightarrow 0)$ emission (adapted from 


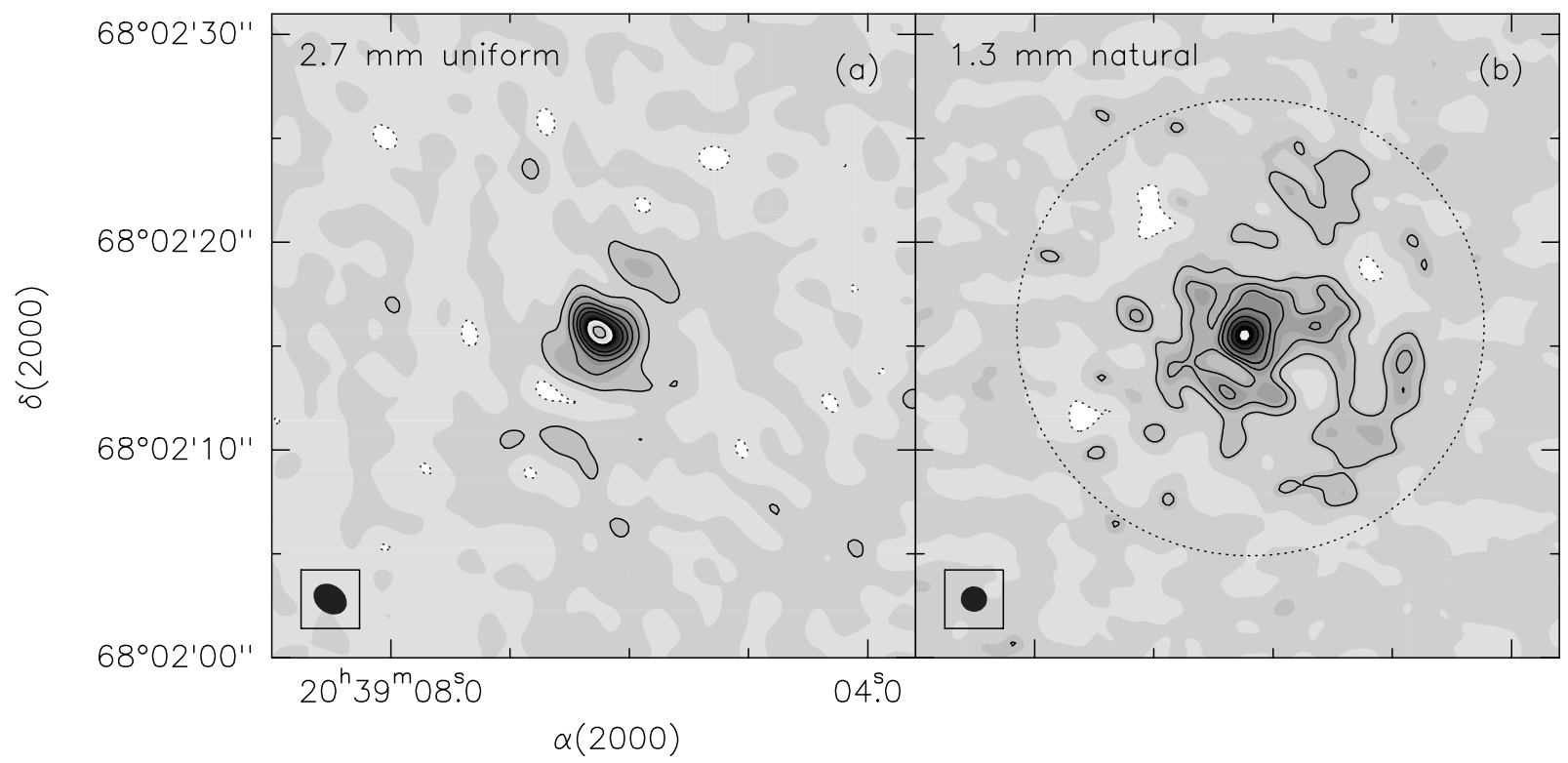

Fig. 1. a) $2.7 \mathrm{~mm}$ continuum emission uniform-weight map. The synthesized beam is $1^{\prime \prime} .70 \times 11^{\prime \prime} 33$ at PA $=38^{\circ}$, and is drawn in the bottom left corner. The rms noise of the map is $0.33 \mathrm{mJy}_{\text {beam }}{ }^{-1}$. The contour levels are $-1,1,3,5,7,9,13$, and $17 \mathrm{mJy} \mathrm{beam}^{-1}$. b) $1.3 \mathrm{~mm} \mathrm{continuum}$ natural-weight map after adding the single-dish data. The synthesized beam is $1^{\prime \prime} .24 \times 1^{\prime \prime} .22$ at PA $=27^{\circ}$, and is drawn in the bottom left corner. The rms noise of the map is $1.4 \mathrm{mJy}_{\text {beam }}{ }^{-1}$. The contour levels are $-5,5,10,15,20,30,40$ and $50 \mathrm{mJy} \mathrm{beam}^{-1}$. The circle represents the Plateau de Bure primary beam (50\% attenuation level).

Table 1. Properties of the compact and extended continuum emission.

\begin{tabular}{|c|c|c|c|c|c|c|c|c|c|}
\hline \multirow[b]{2}{*}{ Component } & \multicolumn{3}{|c|}{ Integrated fluxes } & \multicolumn{3}{|c|}{ Source size ${ }^{c}$} & \multicolumn{2}{|c|}{ Spectral indexes } & \multirow{2}{*}{$\begin{array}{c}\text { Masses } \\
\left(M_{\odot}\right)\end{array}$} \\
\hline & $\begin{array}{c}2.7 \mathrm{~mm}^{a} \\
(\mathrm{mJy})\end{array}$ & $\begin{array}{c}2.7 \mathrm{~mm}^{b} \\
(\mathrm{mJy})\end{array}$ & $\begin{array}{c}1.3 \mathrm{~mm}^{b} \\
(\mathrm{mJy})\end{array}$ & $\left({ }^{\prime \prime}\right)$ & $(\mathrm{AU})$ & PA & $\alpha$ & $\beta=\alpha-2$ & \\
\hline COMPACT & 35 & $25^{d}$ & $78^{e}$ & $1.05 \times 0.58^{f}$ & $\sim 450 \times 250$ & $12^{\circ}$ & $\sim 2.1$ & 0.1 & $\sim 0.12$ \\
\hline EXTENDED & $\sim 60$ & $\sim 31$ & $\sim 680$ & $\sim 8 \times 7$ & $\sim 3500 \times 3100$ & $\sim 155^{\circ}$ & $4-5$ & $2-3$ & $\sim 1.08$ \\
\hline TOTAL $^{g}$ & 95 & 56 & 760 & & & & & & $\sim 1.2$ \\
\hline
\end{tabular}

Peak coordinates of the compact core: $\alpha(\mathrm{J} 2000)=20^{\mathrm{h}} 39^{\mathrm{m}} 06.24^{\mathrm{s}} \delta(\mathrm{J} 2000)=68^{\circ} 02^{\prime} 15.6^{\prime \prime}$. Position accuracy is $0{ }^{\prime} 2$.

(a) Integrated flux density measured by Gueth et al. (1997).

(b) Integrated flux density measured in this paper, corrected for primary beam response.

(c) Measured at $1.3 \mathrm{~mm}$.

(d) Integrated flux density measured by fitting an elliptical Gaussian directly to the visibility data for baselines $\geq 80 \mathrm{~m}$.

(e) Integrated flux density measured by fitting an elliptical Gaussian directly to the visibility data for baselines $\geq 40 \mathrm{~m}$.

( $f$ ) Size obtained by fitting an elliptical Gaussian directly to the visibility data. The uncertainty in the size values is $\sim 0$.'04.

(g) Compact + extended components.

Gueth et al. 1996), which is tracing the molecular outflow, with the $2.7 \mathrm{~mm}$ and $1.3 \mathrm{~mm}$ natural-weight maps (as well as with the $\mathrm{HC}_{3} \mathrm{~N}$ and $\mathrm{C}^{18} \mathrm{O}$ integrated emission). The continuum dust emission is resolved at both wavelengths and clearly shows two components, a compact source plus an extended envelope. In Table 1, we summarize the observed properties of these two components. The position found for the compact source L1157-mm at both wavelengths is $\alpha(\mathrm{J} 2000)=20^{\mathrm{h}} 39^{\mathrm{m}} 06^{\mathrm{s}} .24$, $\delta(\mathbf{J} 2000)=68^{\circ} 02^{\prime} 15^{\prime \prime} 6$. Position accuracy is about 0 ' $^{\prime} 2$. This is in agreement with the position found by Gueth et al. (1996) through previous $115 \mathrm{GHz}$ observations. The position obtained by Gueth et al. (1997) is slightly different, but this was traced back to to an incorrect value for the coordinates of the phase calibrator $2013+370$.

\section{1. $2.7 \mathrm{~mm}$ continuum}

The $2.7 \mathrm{~mm}$ uniform-weight map (Fig. 1) marginally resolves the compact component: the central source is slightly elongated perpendicular to the outflow direction, but this elongation is however dominated by the beam. The deconvolved size we found after fitting an elliptical Gaussian to the uniform-weight map is $\sim 1^{\prime \prime}$. $3 \times 1^{\prime \prime}$. 2 at PA $\simeq 50^{\circ}$, which is different from the position angle of the uniform-weight synthesized beam $\left(\mathrm{PA}=38^{\circ}\right)$. Fitting an elliptical Gaussian directly to the visibility data, for baselines longer than $80 \mathrm{~m}$, we found a total flux of $25 \mathrm{mJy}$ and a size of $1^{\prime \prime} .2 \pm 00^{\prime} 04 \times 00^{\prime} 9 \pm 00^{\prime} 05$ at $\mathrm{PA}=50^{\circ}$. This size, which corresponds to a linear size of $\sim 500 \times 400 \mathrm{AU}$ at the distance of the source, is consistent with the previous determination from 


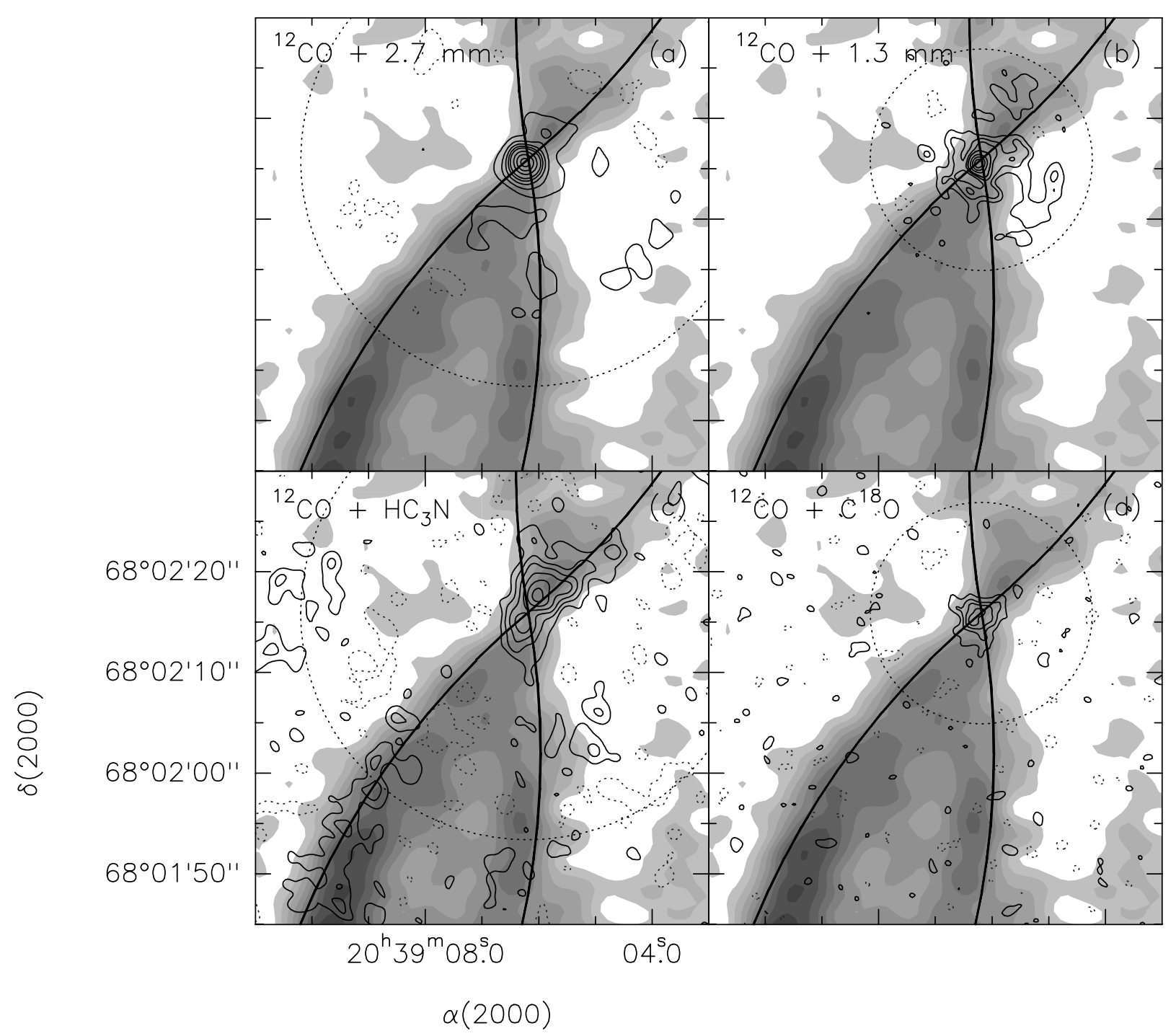

Fig. 2. Overlay of the ${ }^{12} \mathrm{CO}(J=1 \rightarrow 0)$ integrated emission (greyscale; adapted from Gueth et al. 1996) and a) the $2.7 \mathrm{~mm}$ continuum natural-weight map toward L1157 (contours). The rms noise of the map is 0.30 mJy beam $^{-1}$. The contour levels are $-1,1,3,5,7,9,13,17$, and $21 \mathrm{mJy}^{-1}$; $\left.\mathbf{b}\right)$ the $1.3 \mathrm{~mm}$ continuum natural-weight map after adding the single-dish data (contours). The rms noise of the map is $1.4 \mathrm{mJy}_{\text {beam }}{ }^{-1}$. The contour levels are $-5,5,10,15,20,30,40$ and $\left.50 \mathrm{mJy}_{\text {beam }}^{-1} ; \mathbf{c}\right)$ the $\mathrm{HC}_{3} \mathrm{~N}(\mathrm{~J}=12 \rightarrow 11)$ integrated emission $($ contours $)$. The rms noise of the map is $4.8 \mathrm{mJy}_{\text {beam }}^{-1} \mathrm{~km} \mathrm{~s}^{-1}\left(0.11 \mathrm{~K} \mathrm{~km} \mathrm{~s}^{-1}\right)$. The contours are $-12,12,24,36,48,60$ and $72 \mathrm{mJy} \mathrm{beam}^{-1} \mathrm{~km} \mathrm{~s}^{-1}$; and d) the $\mathrm{C}^{18} \mathrm{O}(J=2 \rightarrow 1)$ integrated emission (contours). The rms noise of the map is 16 mJy beam ${ }^{-1} \mathrm{~km} \mathrm{~s}^{-1}\left(0.28 \mathrm{~K} \mathrm{~km} \mathrm{~s}^{-1}\right)$. The contours are $-70,70,140,210$, and $280 \mathrm{mJy}$ beam ${ }^{-1} \mathrm{~km} \mathrm{~s}^{-1}$. The $\mathrm{HC}_{3} \mathrm{~N}$ and the $\mathrm{C}^{18} \mathrm{O}$ emission have been integrated over the outflow velocity range 1.8 to $3.6 \mathrm{~km} \mathrm{~s}^{-1}$. The black lines outline the edges of the ${ }^{12} \mathrm{CO}$ outflow, for reference in other figures. The circles represent the Plateau de Bure primary beam $(50 \%$ attenuation level).

Gueth et al. (1997). However, the flux density value that we have found is slightly lower than the value of $35 \mathrm{mJy}$ measured by these authors.

Extended emission at a low 1 to $3 \mathrm{mJybeam}^{-1}$ level is also visible along the outflow axis at PA of $\sim 155^{\circ}$. This emission has a flux density of $\sim 31 \mathrm{mJy}$ integrated over an area of $\sim 200 \operatorname{arcsec}^{2}$. It is less extended and weaker than the emission detected by Gueth et al. (1997) with a similar synthesized beam. Adding the compact component, the total continuum flux at $2.7 \mathrm{~mm}$ is $\sim 56 \mathrm{mJy}$. This is lower than the flux of $\sim 90$ mJy measured by Gueth et al. (1997). These discrepancies reflect the difficulty of absolute flux calibration in the $\mathrm{mm}$ domain, as well as that of low-level extended structures deconvolution - which may crucially depend on the actual $u v$ coverage.

\section{2. $1.3 \mathrm{~mm}$ continuum}

At $1.3 \mathrm{~mm}$, the compact component has a peak intensity of $60 \mathrm{mJybeam}^{-1}$, and is marginally resolved, with a size of $\sim 1^{\prime \prime} .1 \times 0 .{ }^{\prime} 9$. By fitting an elliptical Gaussian to the visibility data for baselines longer than $40 \mathrm{~m}$ (which is consistent with using $80 \mathrm{~m}$ at $2.7 \mathrm{~mm}$ in order to select the same physical scales), we obtained a total flux of $\sim 78 \mathrm{mJy}$, and a size of $1^{\prime \prime} .05 \pm 00^{\prime} 04 \times 00^{\prime} 58 \pm 0$ ' $^{\prime} 04(\sim 450 \times 250 \mathrm{AU})$ at PA $=12^{\circ}$. Note that both the size and orientation of the compact source are 


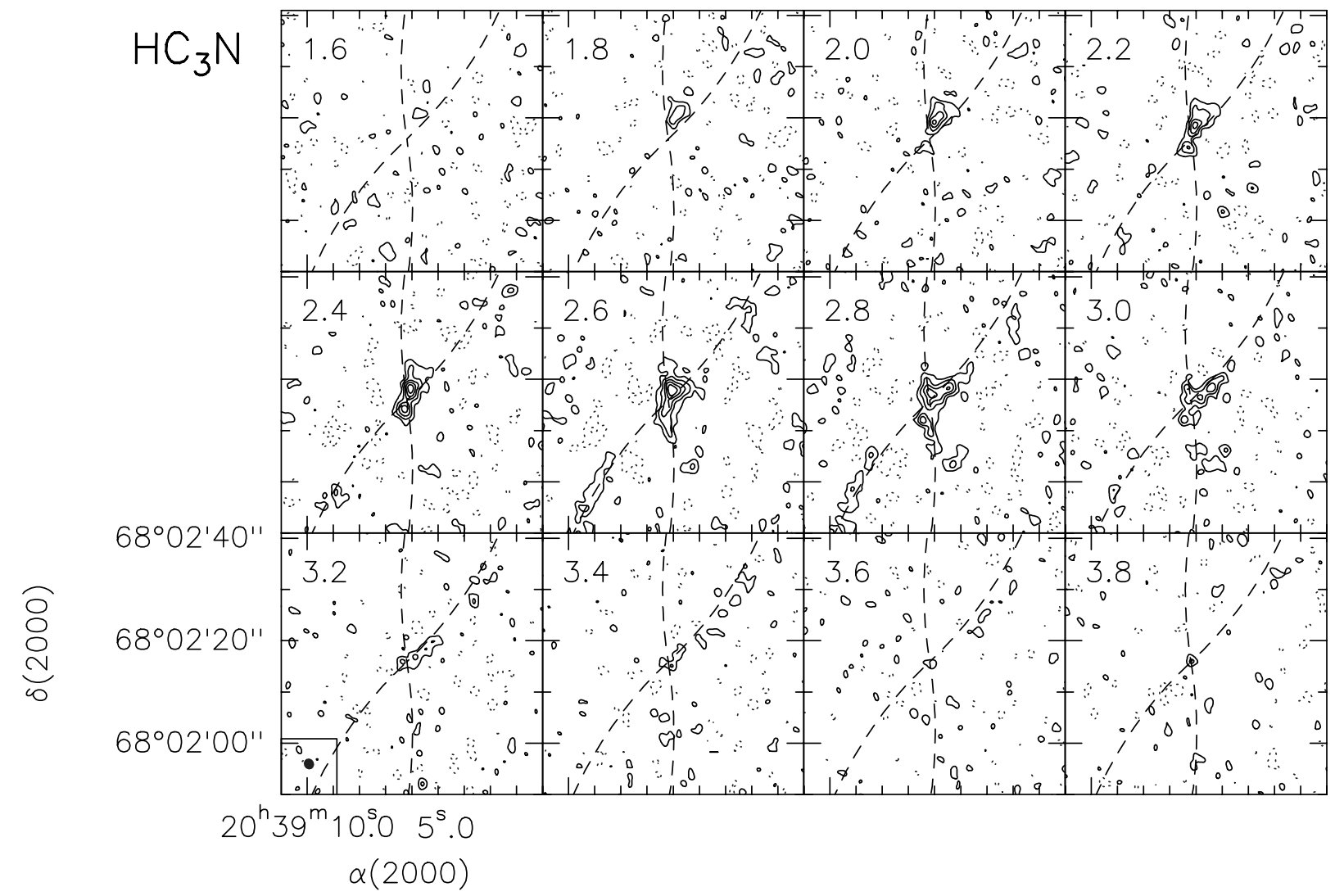

Fig. 3. Velocity channel maps of the $\mathrm{HC}_{3} \mathrm{~N}(J=12 \rightarrow 11)$ emission. The $v_{\mathrm{LSR}}$ of L1157-mm is $2.6 \mathrm{~km} \mathrm{~s}^{-1}$. The central velocity of each velocity interval is indicated at the upper left corner of each panel. The $1-\sigma$ noise in 1 channel is $7.5 \mathrm{mJy} \mathrm{beam}^{-1}$. Contour levels are $-15,15$ to 75 by step of $15 \mathrm{mJy} \mathrm{beam}^{-1}$. The conversion factor from Jy beam ${ }^{-1}$ to $\mathrm{K}$ is 22.5 . The synthesized beam is $2^{\prime \prime} \cdot 24 \times 22^{\prime \prime} 04$ at PA $=33^{\circ}$, and is drawn in the bottom left corner of the bottom left panel. The dash lines outline the edges of the outflow (see Fig. 2).

slightly different at 2.7 and $1.3 \mathrm{~mm}$, which is probably related to the source not having a perfect Gaussian shape structure. In particular, at $1.3 \mathrm{~mm}$ the compact component is slightly elongated along the outflow cavity.

Figures 1 and 2 show that the centrally peaked compact source is surrounded by a structure extended roughly along the flow axis. The flux density is 5 to $15 \mathrm{mJy} \mathrm{beam}^{-1}$ in this component, well above the rms noise, $\sigma \simeq 1.4 \mathrm{mJy} \mathrm{beam}^{-1}$ (estimated over an empty area of the map). We measured a total flux density of $\sim 760 \mathrm{mJy}$ (corrected for primary beam attenuation). This is consistent with the flux of $630 \mathrm{mJy}$ measured at $1.1 \mathrm{~mm}$ by Motte \& André (2001) with MAMBO on the IRAM 30-m telescope. The circumstellar dust emission around L1157-mm is clearly dominated by the extended component. This envelope does not have spherical symmetry, but it is spread over a region of $\sim 8^{\prime \prime}$ ( $\left.\sim 3500 \mathrm{AU}\right)$ along the outflow direction.

The $1.3 \mathrm{~mm}$ map also shows an emission extended in the direction perpendicular to the outflow, on a $\sim 20^{\prime \prime}$ scale. This structure is obviously much too large to be a circumstellar disk. It might be due to a flattened envelope remnant of the molecular cloud in which the protostar was formed. This extended material has also been detected, but at a much larger spatial scale, through $850 \mu \mathrm{m}$ and $1.3 \mathrm{~mm}$ single-dish observations (Shirley et al. 2000; Chini et al. 2001; Gueth et al. 2003).

\section{Molecular line emission}

\section{1. $H C_{3} N$ maps}

The $\mathrm{HC}_{3} \mathrm{~N}(J=12 \rightarrow 11)$ transition has a high critical density $\left(8 \times 10^{5} \mathrm{~cm}^{-3}\right.$, Chung et al. 1991), which makes it an even better tracer of high density regions than e.g. $\mathrm{CS}(J=2 \rightarrow 1)$. Figure 3 shows the velocity channel maps for the $\mathrm{HC}_{3} \mathrm{~N}(J=12 \rightarrow 11)$ emission toward the core of L1157, around the systemic velocity channel $\left(\simeq 2.6 \mathrm{~km} \mathrm{~s}^{-1}\right)$. The gas emission around L1157-mm is not compact, but extended and elongated along the outflow edges. The outflow is blueshifted toward the south and redshifted toward the north and is almost in the plane of the sky (Gueth et al. 1996). As a consequence, there is blueshifted and redshifted $\mathrm{HC}_{3} \mathrm{~N}$ emission in both lobes. The integrated $\mathrm{HC}_{3} \mathrm{~N}$ emission (Fig. 2) has a flux density $\sim 670 \mathrm{mJy} \mathrm{km} \mathrm{s}^{-1}$.

At a distance of $\sim 30^{\prime \prime}$ from the central source, the southern lobe reveals an elongated filament at velocity 2.6 and $2.8 \mathrm{~km} \mathrm{~s}^{-1}$ (Fig. 3). It is outlining very clearly the eastern flank of the outflow $\mathrm{CO}$ lobe. This emission is already significantly affected by the primary beam attenuation. Interestingly, this feature coincides with a SiO shock ( $\mathrm{S} 3$ shock from Gueth et al. 1998; Zhang et al. 2000), traced also by $\mathrm{H}_{2}$ (Davis \& Eislöffel 1995), $\mathrm{NH}_{3}$ (Tafalla \& Bachiller 1995), $\mathrm{H}_{2} \mathrm{CO}, \mathrm{CS}, \mathrm{CH}_{3} \mathrm{OH}$, and SO (Bachiller et al. 2001). Gueth et al. (1996, 1998) and Zhang et al. (2000) describe a precessing episodic jet scenario 


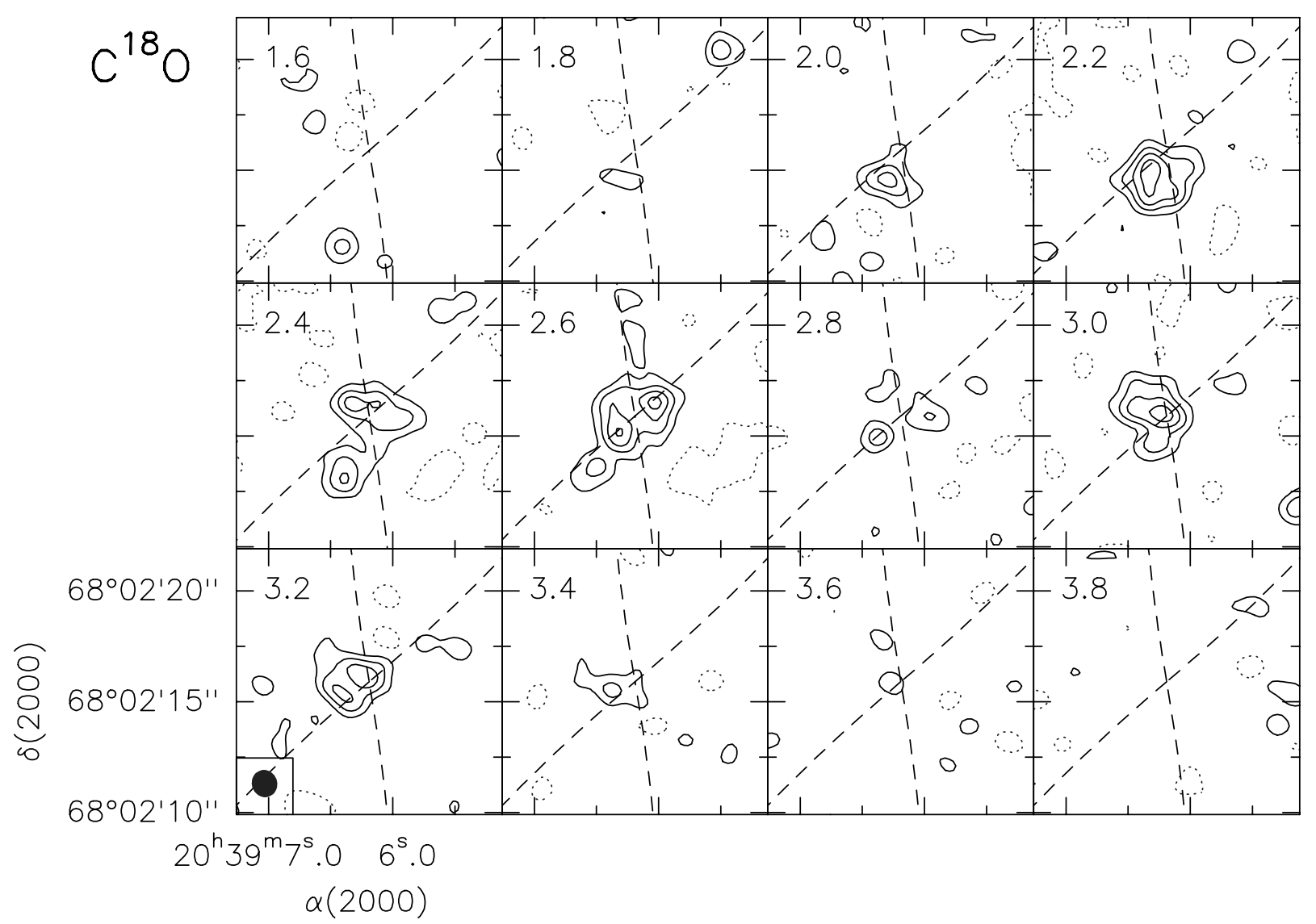

Fig. 4. Velocity channel maps of the $\mathrm{C}^{18} \mathrm{O}(J=2 \rightarrow 1)$ emission. The $v_{\mathrm{LSR}}$ of L1157-mm is $2.6 \mathrm{~km} \mathrm{~s}^{-1}$. The central velocity of each velocity interval is indicated at the upper left corner of each panel. The $1-\sigma$ noise in 1 channel is $30 \mathrm{mJy}$ beam $^{-1}$. Contour levels are $-50,50$ to 200 by step of $50 \mathrm{mJy}_{\text {beam }}{ }^{-1}$. The conversion factor from Jy beam ${ }^{-1}$ to $\mathrm{K}$ is 17.9 . The synthesized beam is $1^{\prime \prime} .24 \times 1^{\prime \prime} .14$ at PA $=71^{\circ}$, and is drawn in the bottom left corner of the bottom left panel. The dash lines outline the edges of the outflow (see Fig. 2).

that can explain the different orientations and velocities of the cavities of the outflow. According to this scenario, the jet has precessed toward the SE-NW direction, and the region discussed here would thus correspond to an event of enhanced ejection, which is impacting the flank of the existing $\mathrm{CO}$ cavity. The resulting shock explains the increased abundances of some species at that location, including $\mathrm{HC}_{3} \mathrm{~N}$ (Bachiller \& Pérez-Gutiérrez 1997). The velocity of the $\mathrm{HC}_{3} \mathrm{~N}$ gas is significantly lower than that of the $\mathrm{CO}$ emission at the same location (e.g. Gueth et al. 1996). This suggests that high velocity material has a lower density, and would thus not be seen in $\mathrm{HC}_{3} \mathrm{~N}$,

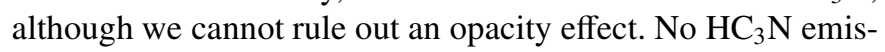
sion has been detected inside the cavity, but an extended emission could possibly have been filtered out by the interferometer.

\section{2. $C^{18} \mathrm{O}$ maps}

Figure 4 presents the velocity channel maps for the $\mathrm{C}^{18} \mathrm{O}(J=2 \rightarrow 1)$ emission. The integrated emission (Fig. 2) appears more compact than the $\mathrm{HC}_{3} \mathrm{~N}$ emission: it is dominated by the central compact component, and is thus associated to the innermost part of the protostellar envelope. The total integrated intensity is $\sim 3 \mathrm{Jy} \mathrm{km} \mathrm{s}^{-1}$. The $\mathrm{C}^{18} \mathrm{O}$ emission is also tracing gas associated to the outflow, as can be seen at the systemic velocity channel map $\left(v_{\mathrm{LSR}}=2.6 \mathrm{~km} \mathrm{~s}^{-1}\right.$, see Fig. 4). A striking feature visible in Fig. 4 is that the $\mathrm{C}^{18} \mathrm{O}$ emission at $2.8 \mathrm{~km} \mathrm{~s}^{-1}$ is weaker than in the previous and following velocity channels. This effect is clearly seen as a narrow deep self-absorption in the spectrum taken at the position of L1157-mm (see below, Sect. 6.2.1 and Fig. 6).

\section{Analysis}

\subsection{Spectral index map}

Figure 5 presents a map of the spectral index $\alpha$, where $S_{v} \propto v^{\alpha}$, computed using the 2.7 and $1.3 \mathrm{~mm}$ continuum interferometric images restored with the same resolution, 2".2 (or 900 AU). The spectral index varies from a value $\sim 2.1$, at the position of the compact source to a value of $\sim 4-5$ at larger distance ${ }^{1}$. Instrumental effects such as missing flux and/or a wrong flux scale would affect the absolute level of the spectral index map, but can hardly introduce a spatial variation. Line contamination can be excluded, since it does not affect the interferometer data and was found to be absent in the bolometer measurement (Gueth et al. 2003). The observed variation accross the

${ }^{1}$ Gueth et al. (2003) have analyzed the spectral index around L1157-mm from bolometric observations at 1.2 and $0.85 \mathrm{~mm}$. They obtained an intermediate value of $\alpha \simeq 3.5$ in the central $20^{\prime \prime}$ beam which includes all our interferometric map. 


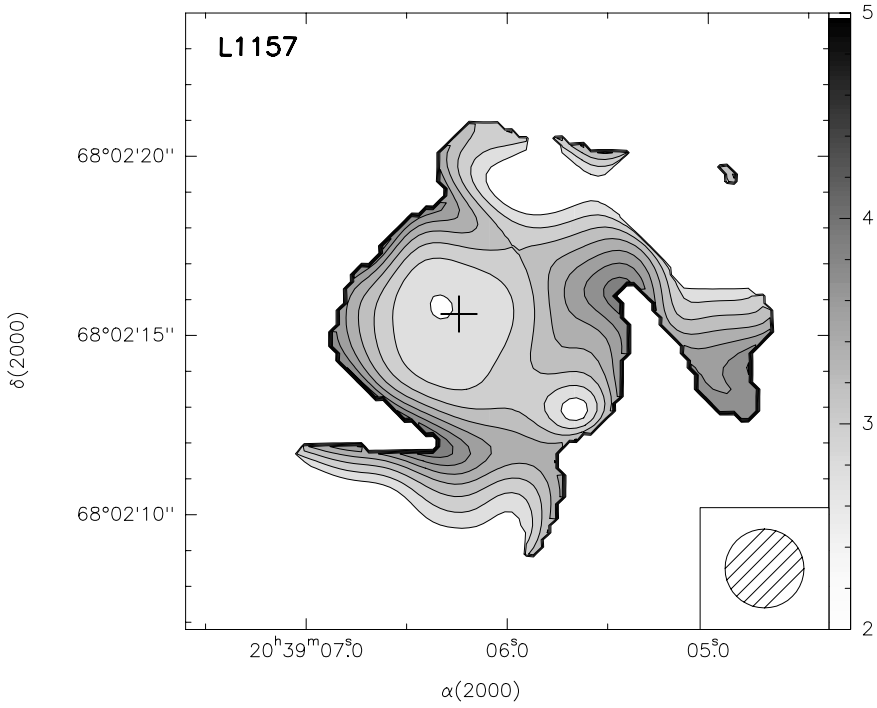

Fig. 5. Spectral index map from the 2.7 and $1.3 \mathrm{~mm}$ continuum images, restored to the same resolution of 2 '" 2 . Contour levels are plotted from 2 to 5 by intervals of 0.5 . Greyscale is linear in the range of $2-5$, and shows the regions with higher spectral index darker. The cross marks the position of L1157-mm.

L 1157-mm region may thus be indicative of physical differences between the properties of the material toward the embedded source and those of the more extended region. This effect was already observed in other young embedded sources (Visser et al. 1998; Johnstone \& Bally 1999; Smith et al. 2000; Beltrán et al. 2002).

A low value of $\alpha$, as observed at the central position, can be due to optically thick dust emission. In such a case, the dust temperature $T_{\mathrm{d}}$ is equal to the brightness temperature $T_{\mathrm{B}}$ (in the Rayleigh-Jeans approximation), which can be derived from the observed $1.3 \mathrm{~mm}$ flux density:

$S_{v}=\frac{2 k v^{2}}{c^{2}} T_{\mathrm{B}} \Omega$

where $\Omega$ is the solid angle subtended by the source. An upper limit on $T_{\mathrm{d}}$ can be computed when assuming that the whole flux of the compact core (78 mJy, see Table 1) is coming from the optically thick contribution. Assuming that the size of the optically thick emitting region is around $150 \mathrm{AU}$, we derive a value of $T_{\mathrm{d}}<10 \mathrm{~K}$ for the dust temperature (note that the larger the optically thick core, the lower $T_{\mathrm{d}}$ ). This value is very low and suggests that a significant fraction of the compact core has an optically thin emission.

Assuming that the dust emission at mm wavelengths is optically thin and in the Rayleigh-Jeans regime, $\alpha$ is related to the power-law index $\beta$ of the dust emissivity $\kappa_{v} \propto v^{\beta}$, through $\alpha=\beta+2$. For the more extended material, $\beta$ would thus be larger than 2 , the typical value for interstellar dust grains (see e.g. Draine $\&$ Lee 1984), while the value of $\beta$ for the compact source would be $\sim 0.1$. Interestingly, this decrease of $\beta$ could be due to larger grains (e.g. Mannings \& Emerson 1994), hence suggesting grain growth in the denser inner regions. Grain shape evolution or chemical evolution are also possible (see, e.g. Ossenkopf \& Henning 1994; Pollack et al. 1994).

\subsection{Envelope mass - from continuum emission}

Assuming optically thin dust thermal emission, the mass of the emitting material can be derived from the $1.3 \mathrm{~mm}$ continuum flux density $S_{v}$ by:

$M=\frac{S_{v} D^{2}}{\kappa_{v} T} \frac{c^{2}}{2 k v^{2}}$.

Using a dust temperature of $40 \mathrm{~K}$ (following Gueth et al. 1997), and a dust mass opacity of $0.01 \mathrm{~cm}^{2} \mathrm{~g}^{-1}$, as recommended by Henning et al. (1995) for a gas-to-dust ratio of 100, we obtain a mass of the central compact source of $\sim 0.12 M_{\odot}$, while the mass of the envelope is $\sim 1.08 M_{\odot}$. Thus, the total (compact + extended) circumstellar mass is $\sim 1.2 M_{\odot}$. This is a factor 3 below the value of $\sim 3 M_{\odot}$ derived by Gueth et al. (1997) at $2.7 \mathrm{~mm}$. The absolute flux density of the $1.3 \mathrm{~mm}$ data may be significantly underestimated. These calculations are also dependent on the dust opacity law used, the mass being lower in case of higher dust opacity. In any case, the large ratio between the mass of the extended component and that of the compact one indicates that there is an important reservoir of material that can be incorporated into the central protostar. This is in agreement with the conceptual definition of a Class 0 object.

\subsection{Envelope mass - from gas emission}

The gas mass of the protostellar condensation can be better estimated from the $\mathrm{C}^{18} \mathrm{O}$ data, since the $\mathrm{HC}_{3} \mathrm{~N}$ emission is partly associated to the outflow. Following the derivation of Scoville et al. (1986), and assuming optically thin emission, the $\mathrm{C}^{18} \mathrm{O}$ beam averaged column density is given by

$\bar{N}=1.15 \times 10^{13} \frac{\left(T_{\text {ex }}+0.88\right)}{\mathrm{e}^{-15.81 / T_{\text {ex }}}} \int T_{B} \mathrm{~d} v \mathrm{~cm}^{-2}$

where $T_{\mathrm{ex}}$ is the excitation temperature, and $\int T_{\mathrm{B}} \mathrm{d} v$ is the integrated brightness temperature of the $\mathrm{C}^{18} \mathrm{O}(J=2 \rightarrow 1)$ emission. Assuming $T_{\mathrm{ex}}=40 \mathrm{~K}$, a $\mathrm{C}^{18} \mathrm{O}$ abundance relative to molecular hydrogen of $1.4 \times 10^{-7}$, and a mean molecular weight of $2.6 m_{\mathrm{H}}$, we obtain a mass of $\sim 9 \times 10^{-3} M_{\odot}$ in the inner $\sim 2^{\prime \prime} \times$ $2^{\prime \prime}$ area (roughly corresponding to the central source). It should be mentioned that the excitation temperature value adopted does not affect significantly the estimated molecular mass; the mass is $\sim 7 \times 10^{-3} M_{\odot}$ for $T_{\mathrm{ex}}=20 \mathrm{~K}$, and $\sim 11 \times 10^{-3} M_{\odot}$ for $T_{\text {ex }}=50 \mathrm{~K}$. Our derived value is certainly a lower limit since the $\mathrm{C}^{18} \mathrm{O}$ is affected by self-absorption (see Sect. 6.2.1), and hence partly optically thick. Indeed, Gueth et al. (1997) derived a mass of $4.7 \times 10^{-2} M_{\odot}$ from $\mathrm{C}^{18} \mathrm{O}(J=1 \rightarrow 0)$. Comparing the molecular mass, a few $10^{-2} M_{\odot}$, to that derived from the continuum data, $M \simeq 0.12 M_{\odot}$, it suggests that $\mathrm{CO}$ in L1157-mm could be depleted by a factor around $\sim 3-10$, although this result has to be taken with caution in case the gas is optically thick and/or clumpy. Using the same assumptions as above, the mass of the whole $\mathrm{C}^{18} \mathrm{O}$ envelope $\left(\sim 8^{\prime \prime} \times 8^{\prime \prime}\right.$ area $)$ is $\sim 4 \times 10^{-2} M_{\odot}$, again significantly below the mass derived from the continuum emission $\left(1.2 M_{\odot}\right)$. However, the $\mathrm{C}^{18} \mathrm{O}$ emission is less extended that the continuum emission, and the comparison between the two masses may therefore not be relevant. 


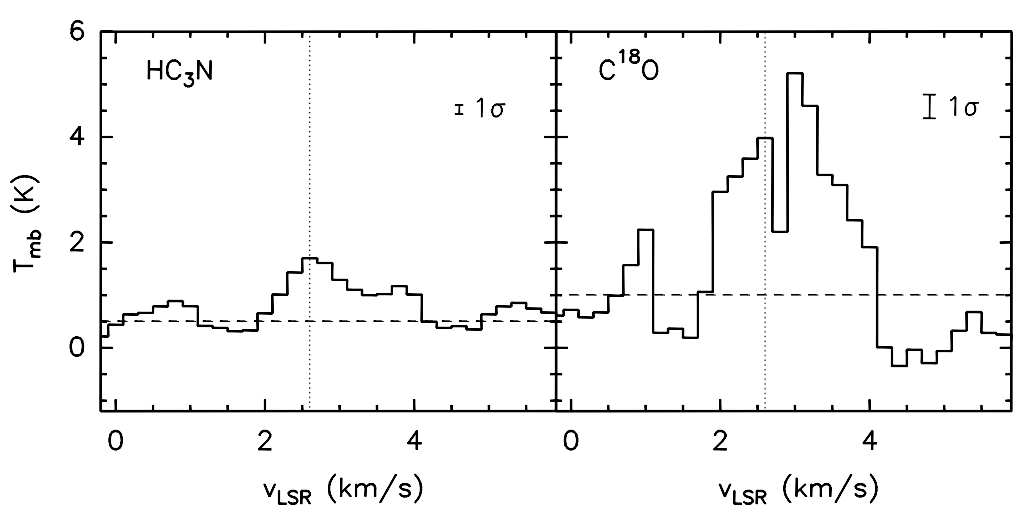

Fig. 6. $\quad \mathrm{HC}_{3} \mathrm{~N} \quad(J=12 \rightarrow 11) \quad$ (left) and $\mathrm{C}^{18} \mathrm{O}(J=2 \rightarrow 1)$ (right) spectra obtained at the position of L1157$\mathrm{mm}$. The continuum emission has not been subtracted (the continuum level is indicated by the dashed horizontal line). The dashed vertical line indicates the $v_{\mathrm{LSR}}$ of $2.6 \mathrm{~km} \mathrm{~s}^{-1}$. The $1-\sigma$ noise in 1 channel is $0.17 \mathrm{~K}$ for $\mathrm{HC}_{3} \mathrm{~N}$, and $0.45 \mathrm{~K}$ for $\mathrm{C}^{18} \mathrm{O}$. The conversion factor of $\mathrm{Jy}_{\text {beam }}{ }^{-1}$ to $\mathrm{K}$ is 22.5 for $\mathrm{HC}_{3} \mathrm{~N}$, and 17.9 for $\mathrm{C}^{18} \mathrm{O}$.
It is also possible that we miss some $\mathrm{C}^{18} \mathrm{O}(J=2 \rightarrow 1)$ emission because of the lack of short spacings in our measurement.

\section{Discussion}

\subsection{Interaction between the outflow and the envelope}

Both our continuum and lines observations reveal structures or filaments that seem to be associated with the edges of the L 1157 outflow.

The $1.3 \mathrm{~mm}$ continuum map (Fig. 2) reveals a low-level extended emission whose conical shape matches that of the outflow lobes. Gueth et al. (1997) reported a similar feature from $2.7 \mathrm{~mm}$ observations: the dust emission was observed and modelled as a "cross-like" structure coincident with the outflow edges. Note however that these observations indicated opening angles slightly smaller than those shown by our maps. The morphological association between the outflow edges and dense protostellar material in L 1157 is even better shown by the $\mathrm{HC}_{3} \mathrm{~N}$ observations. The channel maps presented in Fig. 3 (see the 2.6 to $3.2 \mathrm{~km} \mathrm{~s}^{-1}$ channels) reveal extensions that outline quite accurately the beginning of the outflow conical lobes. The southeastern arm of this "cross-like" pattern is missing, as in the $2.7 \mathrm{~mm}$ continuum from Gueth et al. (1997), which suggests different temperature and/or density than in the other arms. The $\mathrm{C}^{18} \mathrm{O}$ emission, while much more compact than the $\mathrm{HC}_{3} \mathrm{~N}$ emission, exhibits marginal indications of a similar morphology.

Following Gueth et al. (1997), we suggest that these morphological coincidences indicate that a strong interaction between the outflow and the protostellar envelope is taking place in L 1157: the outflow sweeps up envelope material, thereby creating heated/compressed regions at its edges, which, observationaly, mimic a "cross-like" structure (see Gueth et al. 1997, their Fig. 10). Circumstellar dust and gas material around young stellar objects outlining the edges of the molecular outflow have also been mapped in other sources (e.g. B5-IRS1: Langer et al. 1996; L1527: Ohashi et al. 1997; Hogerheijde et al. 1998; Motte \& André 2001; L1551-IRS5: Fuller et al. 1995; Ladd et al. 1995).

Interestingly, the $\mathrm{HC}_{3} \mathrm{~N}$ emission also revels two peaks located on both sides of the central protostar. They are clearly visible in the integrated emission map (Fig. 2) as well as in the individual channel maps (Fig. 3). Their positions coincide with the apparent base of the outflow conical cavities as traced by the ${ }^{12} \mathrm{CO}$ (see Fig. 2), suggesting that these emission peaks could be produced when the dense gas is shock-heated and compressed by the flow. Hence, we conclude that, at the resolution of our observations, the structure of the dust+gas protostellar envelope is strongly affected by the presence of the outflow.

\subsection{Kinematics of the gas}

\subsection{1. $\mathrm{C}^{18} \mathrm{O}$ self-absorption}

Figure 6 presents the $\mathrm{HC}_{3} \mathrm{~N} \quad(J=12 \rightarrow 11)$ and $\mathrm{C}^{18} \mathrm{O} \quad(J=2 \rightarrow 1)$ spectra obtained at the position of L1157-mm. As already mentioned, the $\mathrm{C}^{18} \mathrm{O}$ emission shows a narrow $\left(\Delta v \simeq 0.2 \mathrm{~km} \mathrm{~s}^{-1}\right)$ deep absorption feature in the spectra, at a velocity of $\sim 2.8 \mathrm{~km} \mathrm{~s}^{-1}$. A similar feature of $\sim 0.3 \mathrm{~km} \mathrm{~s}^{-1}$ was also reported at the same velocity in the interferometric ${ }^{13} \mathrm{CO}(J=1 \rightarrow 0)$ map and spectra by Gueth et al. (1997). This suggests that the $C^{18} \mathrm{O}(J=2 \rightarrow 1)$ line is selfabsorbed. However, missing short-spacings information could cause a fake absorption, but spatially extended (see Gueth et al. 1997, their Fig.7). In Fig. 7 we plot the $\mathrm{C}^{18} \mathrm{O}(J=2 \rightarrow 1)$ spectra around L1157-mm, by offsets of 1. .'2, roughly the size of our synthesized beam. This figure shows that the absorption feature is only detected at a few positions, close to the compact source and not at larger distances. Hence, the self-absorption, confined in a small region $\left(\leq 5^{\prime \prime}\right.$ or $\left.\sim 2000 \mathrm{AU}\right)$ around L1157-mm, appears real. Note that Fig. 7 also shows line broadening localized toward the center.

A redshifted self-absorption is naturally explained in a scenario where an optically thick line is observed in an infalling core with temperature decreasing outward (e.g. Leung $\&$ Brown 1977). Figure 6 shows that the brightness temperature (in $T_{\mathrm{mb}}$ scale) of the self-absorbed feature is $\sim 2.2 \mathrm{~K}$. Assuming optically thick emission, it corresponds to an excitation temperature of $T_{\mathrm{ex}} \simeq 6.3 \mathrm{~K}$, in excellent agreement with the excitation temperature found by Gueth et al. (1997) from the ${ }^{13} \mathrm{CO}$ spectra. This low $T_{\mathrm{ex}}$ indicates that the self-absorption features are most probably sub-thermally excited, and should thus be produced in a medium whose density is low enough to prevent thermalization of the lines. No self-absorption was detected in the $\mathrm{C}^{18} \mathrm{O}(J=1 \rightarrow 0)$ interferometric maps obtained by Gueth et al. (1997). This suggests that the $(J=1 \rightarrow 0)$ line is optically thin while the $(J=2 \rightarrow 1)$ is significantly thicker. For $T_{\mathrm{ex}}=10 \mathrm{~K}$, the ratio of optical depths between the two 


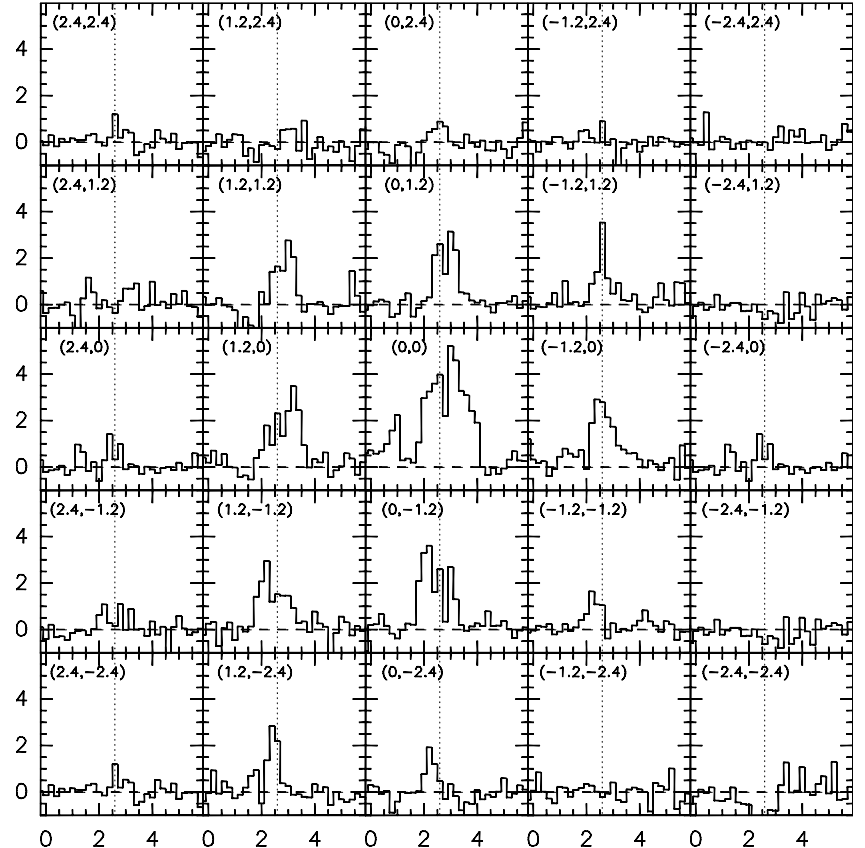

Fig. 7. $\mathrm{C}^{18} \mathrm{O}(J=2 \rightarrow 1)$ spectra obtained around the L1157-mm central region. The continuum has not been subtracted from the line emission. Offsets from the central position are indicated (in ") at the upper left corner of each plot. The dashed vertical line indicates the $v_{\mathrm{LSR}}$ of $2.6 \mathrm{~km} \mathrm{~s}^{-1}$.

transitions is $\tau(J=2 \rightarrow 1) \simeq 1.9 \tau(J=1 \rightarrow 0)$. Note also that the $\mathrm{C}^{18} \mathrm{O}(J=1 \rightarrow 0)$ spectra from Gueth et al. (1997) has a narrow profile, making it difficult to detect a possible asymetry.

\subsubsection{Other absorption features?}

In addition to the self-absorption feature discussed in the previous paragraph, Fig. 6 reveals that both the $\mathrm{HC}_{3} \mathrm{~N}$ and $\mathrm{C}^{18} \mathrm{O}$ spectra at the position of L $1157-\mathrm{mm}$ have weaker emission in the 1-2 $\mathrm{km} \mathrm{s}^{-1}$ (blueshifted) and the $4-5 \mathrm{~km} \mathrm{~s}^{-1}$ (redshifted) velocity intervals. This suggests that the actual line profile may be triangular-shaped, with two strong absorption features. In both lines, each of the "absorbed" channels is at a few $\sigma$ below the un-affected emission, but the whole absorption feature is significant because it extends over 3 to 6 adjacent channels. The redshifted feature in the $\mathrm{C}^{18} \mathrm{O}$ even shows an aborption of the continuum emission.

To explain such absorptions in the spectra, one has to invoke the presence of cold absorbing gas in front of a warm emitting region. Concerning the blueshifted feature, it could arise from self-absorption in the blueshifted southern outflow lobe (see Fig. 8): cold gas at the cavity edge is located in front of warmer material present along the flow axis; the latter gas is expected to have larger velocities, but since it is closer to the plane of the sky, projection effects may produce the same lineof-sight velocity for both components. As for the redshifted absorption feature, a similar mechanism in the redshifted, northern lobe cannot be proposed because the cold material would be located behind the warm gas (Fig. 8). Moreover, any phenomenum in the redshifted outflow lobe could not explain the absorption of the continuum, since this lobe is behind the

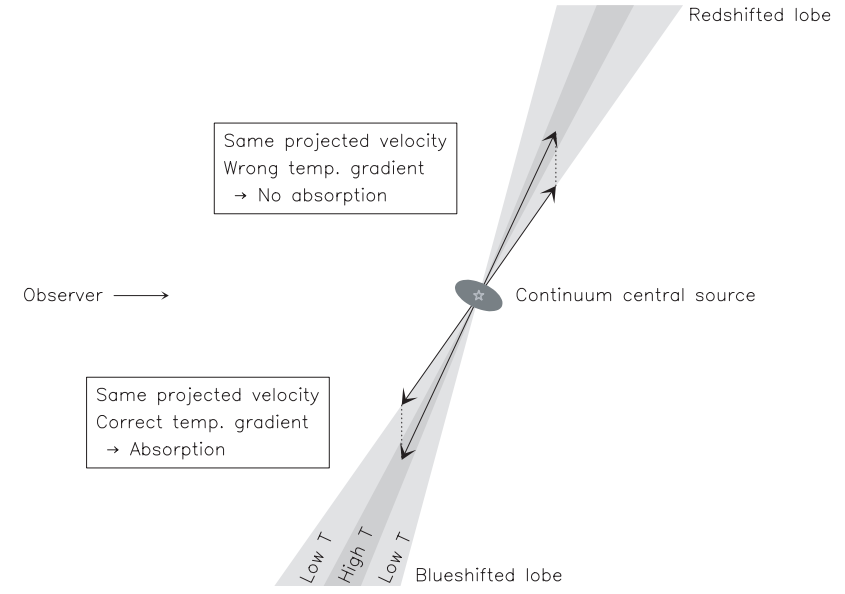

Fig. 8. Geometry of the disk/outflow system in L 1157 and possible origin of the blueshifted absorption feature.

central source (which is at the origin of the compact continuum emission). Instead, one could invoke absorption by the infalling envelope, whose redshifted part is in front of the continuum source.

This latter scenario can drive interesting constrains on the size of the continuum emitting region. The velocity at which the redshifted continuum absorption is observed is higher than the self-absorption feature: this means than the absorbing gas is located closer to the center, and thus should be hotter. The excitation temperature should thus be higher than $6.3 \mathrm{~K}$. On another hand, the excitation temperature must be significantly smaller than the continuum brightness temperature $(1 \mathrm{~K})$, in order to create an absorption. These constrains can be reconciled if we assume that the continuum emission is dominated by a strongly peaked central component, which is affected by beam dillution effects. Let's assume that the $\mathrm{C}^{18} \mathrm{O}$ absorbing material has an excitation temperature of $10 \mathrm{~K}$. Judging from the absorption feature (Fig. 6), the continuum true brightness temperature must be typically $\sim 5$ times larger, i.e. $\sim 50 \mathrm{~K}$. This can be explained by a beam dilution of $\sim 50$ in area, hence $\sim 7$ in size. This in turn would imply a continuum source size of $\sim 75 \mathrm{AU}$ (beamsize/7 $\simeq 0$ ' 17 ). Interestingly, this value is of the order of the expected typical size of a circumstellar disk in a Class 0 object (e.g. Pudritz et al. 1996).

Clearly, these absorption features have to be confirmed and would deserve further, more detailed, observations in order to derive a clear understanding of the complex kinematics of the L1157 protostellar envelope.

\subsubsection{Rotation?}

It should be mentioned that we did not find any evidence of velocity gradient for the $\mathrm{C}^{18} \mathrm{O}(J=2 \rightarrow 1)$ transition (see Fig. 4), indicative of rotation, as suggested by Gueth et al. (1997) for the $\mathrm{C}^{18} \mathrm{O}(J=1 \rightarrow 0)$ line.

\section{Summary}

We have studied with the IRAM Plateau de Bure millimeter interferometer the dust and gas emission toward 
the core of the Class 0 object L1157-mm. Our main conclusions are:

- The continuum emission at 2.7 and $1.3 \mathrm{~mm}$ show a compact component which is resolved at $1.3 \mathrm{~mm}$ into a flattened compact core of $\sim 450 \times 250 \mathrm{AU}$, and mass $\sim 0.12 M_{\odot}$ and is surrounded by an extended envelope of $\sim 3000 \mathrm{AU}$, and mass $\sim 1.1 M_{\odot}$.

- The millimeter spectral index varies across the region. A lower value $\alpha \simeq 2.1$ is found toward the position of the compact protostar, while the spectral index is $\alpha \geq 4$ for the extended surrounding material. These values imply a dust emissivity index $\beta \simeq 0.1$ and $\beta \geq 2$, respectively. This variation could possibly indicate grain growth toward the compact component.

- A strong interaction between the molecular outflow and the close protostellar environment is taking place and affects the structure of the innermost parts of the envelope. This is shown by the spatial correlation between the molecular outflow and the dust continuum (1.3 mm map) and the $\mathrm{HC}_{3} \mathrm{~N}$ emission: both maps show structures associated to the edges of the outflow lobes, as traced by the ${ }^{12} \mathrm{CO}$ emission.

- $\mathrm{HC}_{3} \mathrm{~N}$ emission is also detected at a distance of $\sim 13000 \mathrm{AU}$ from the central object, at the position of a shock associated to the impact of the precessing jet against the walls of the main cavity of the southern lobe.

- Evidence of infall has been detected through the $\mathrm{C}^{18} \mathrm{O}(J=2 \rightarrow 1)$ observations, in agreement with the indications of infall detected through ${ }^{13} \mathrm{CO}$ by Gueth et al. (1997)

- We did not detect any velocity gradient indicative of a rotating circumstellar disk.

Finally, when observing Class 0 environment such as that of L 1157-mm, multiple velocity components and temperature gradients along the line of sight do confuse the interpretation of spectral line emission. In particular, they make it difficult to disentangle the various components (disk, envelope, outflow) and determine their velocity distributions. Our results show that even the highest density gas tracers such as $\mathrm{HC}_{3} \mathrm{~N}$ are affected by the ejection phenomena, tracing also ambient material compressed by the outflow. When deriving results from low angular resolution observations of Class 0 objects one should be aware of these problems. High angular resolution observations of different dust and gas tracers are absolutely necessary to get a detailed and accurate picture of such young objects environment.

Acknowledgements. We would like to thank Paul Ho for his valuable comments on this work. We acknowledge the IRAM staff from the Plateau de Bure for carrying the observations. M.T.B. acknowledges support from a SAO predoctoral fellowship.

\section{References}

Adams, F. C., Lada, C. J., \& Shu, F. H. 1987, ApJ, 312, 788

Avery, L. W., \& Chiao, M. 1996, ApJ, 463, 642

Bachiller, R., Liechti, S., Walmsley, C. M., \& Colomer, F. 1995, A\&A, 295, L51
Bachiller, R., Martín-Pintado, J., \& Fuente, A. 1993, ApJ, 417, L45

Bachiller, R., \& Pérez Gutiérrez, M. 1997, ApJ, 487, L93

Bachiller, R., Pérez Gutiérrez, M., Kumar, M. S. N., \& Tafalla, M. 2001, A\&A, 372, 899

Beltrán, M. T., Estalella, R., Ho, P. T. P., et al. 2002, ApJ, 565, 1069

Cassen, P., \& Moosman, A. 1981, Icarus, 48, 353

Chini, R., Ward-Thompson, D., Kirk, J. M., et al. 2001, A\&A, 369, 155

Chung, H. S., Osamu, K., \& Masaki, M. 1991, JKAS, 24, 217

Davis, C. J., \& Eislöffel, J. 1995, A\&A, 330, 851

Draine, B. T., \& Lee, H. M. 1984, ApJ, 285, 89

Fuller, G. A., Ladd, E. F., Padman, R., Myers, P. C., \& Adams, F. C. 1995, ApJ, 454, 862

Gueth, F., Guilloteau, S., \& Bachiller, R. 1996, A\&A, 307, 891

Gueth, F., Guilloteau, S., \& Bachiller, R. 1998, A\&A, 333, 287

Gueth, F., Guilloteau, S., Dutrey, A., \& Bachiller, R. 1997, A\&A, 323, 943

Gueth, F., Bachiller, R., \& Tafalla, M. 2003, A\&A, 401, L5

Guilloteau, S., Delannoy, J., Downes, D., et al. 1992, A\&A, 262, 624

Henning, Th., Michel, B., \& Stognienko, R. 1995, P\&SS, 43, 1333

Heyvaerts, J., \& Norman, C. 1989, ApJ, 347, 1055

Hirano, N., \& Taniguchi, Y. 2001, ApJ, 550, L219

Hodapp, K.-W. 1994, ApJSS, 94, 615

Hogerheijde, M. R. 2001, ApJ, 553, 618

Hogerheijde, M. R., van Dishoeck, E. F., Blake, G. A., \& van Langevelde, H. J. 1998, ApJ, 502, 315

Johnstone, D., \& Bally, J. 1999, ApJ, 510, L49

Königl, A., \& Pudritz R. E. 2000, in Protostars and Planets IV, ed. V. Mannings, A. P. Boss, \& S. S. Russell (Tucson: University of Arizona Press), 759

Ladd, E. F., Fuller, G. A., Padman, R., Myers, P. C., \& Adams, F. C. 1995, ApJ, 439, 771

Lai, S.-P., \& Crutcher, R. M. 2000, ApJS, 128, 271

Langer, W. D., Velusamy, T., \& Xie, T. 1996, ApJ, 468, L41

Larson, R. B. 1969, MNRAS, 145, 271

Leung, C., \& Brown, R. 1977, ApJ, 214, L73

Mannings, V., \& Emerson, J. P. 1994, MNRAS, 267, 361

Motte, F., \& André, P. 2001, A\&A, 365, 440

Ohashi, N., Hayashi, M., Ho, P. T. P., \& Momose, M. 1997, ApJ, 475, 211

Ohashi, N., Lee, S. W., Wilner, D. J., \& Hayashi, M. 1999, ApJ, 518, L41

Ossenkopf, V., \& Henning, T. 1994, A\&A, 291, 943

Pollack, J. B., Hollenback, D., Beckwith, S. W. V., et al. 1994, ApJ, 421,615

Pudritz, R. E., \& Norman, C. A. 1983, ApJ, 274, 677

Pudritz, R. E., Wilson, C. D., Carlstrom J. E, et al. 1996, ApJ, 470, L123

Saito, M., Kawabe, R., Kitamura, Y., \& Sunada, K. 1996, ApJ, 473, 464

Scoville, N. Z., Sargent, A. I., Sanders, D. B., et al. 1986, ApJ, 303, 416

Shirley, Y. L., Evans, N. J., Rawlings, J. M. C., \& Gregersen, E. M. 2000, ApJS, 131, 249

Shu, F. H., Adams, F. C. \& Lizano, S. 1987, ARA\&A, 25, 23

Shu, F., Najita, J., Ostriker, E., et al. 1994, ApJ, 429, 781

Smith, K. W., Bonnell, I. A., Emerson, J. P., \& Jenness, T. 2000 MNRAS, 319, 991

Tafalla, M., \& Bachiller, R. 1995, ApJ, 443, L37

Terebey, S., Shu, F. H., \& Cassen, P. 1984, ApJ, 286, 529

Umemoto, T., Iwata, T., Fukui, Y., et al. 1992, ApJ, 392, L83

Visser, A. E., Richer, J. S., Chandler, C. J., \& Padman, R. 1998, MNRAS, 301, 585

Zhang, Q., Ho, P. T. P., \& Wright, M. C. H. 2000, AJ, 119, 1345

Zhang, Q., Ho, P. T. P., Wright, M. C. H., \& Wilner, D. J. 1995, ApJ, 451, L71 